\title{
Review
}

\section{Phenotypic plasticity and evolution by genetic assimilation}

\author{
Massimo Pigliucci ${ }^{1, *}$, Courtney J. Murren ${ }^{2}$ and Carl D. Schlichting ${ }^{3}$ \\ ${ }^{1}$ Department of Ecology and Evolution, SUNY-Stony Brook, 650 Life Science Building, Stony Brook NY 11794, USA, \\ ${ }^{2}$ Department of Biology, College of Charleston, Charleston, SC 29424, USA and ${ }^{3}$ Department of Ecology and \\ Evolutionary Biology, University of Connecticut, Storrs, CT 06269, USA \\ *Author for correspondence (e-mail: pigliucci@genotypbyenvironment.org)
}

Accepted 3 January 2006

\begin{abstract}
Summary
In addition to considerable debate in the recent evolutionary literature about the limits of the Modern Synthesis of the 1930s and 1940s, there has also been theoretical and empirical interest in a variety of new and not so new concepts such as phenotypic plasticity, genetic

misunderstandings, including unwarranted fears of a general attempt at overthrowing the Modern Synthesis paradigm, and some fundamental conceptual confusion about the proper roles of phenotypic plasticity and natural selection within evolutionary theory.
\end{abstract} assimilation and phenotypic accommodation. Here we consider examples of the arguments and counterarguments that have shaped this discussion. We suggest that much of the controversy hinges on several

Key words: phenotypic plasticity, genetic assimilation, phenotypic accommodation, Modern Synthesis, natural selection, evolution.

\section{Introduction}

Why revisit a 50 year old debate?

Genetic assimilation is a process whereby environmentally induced phenotypic variation becomes constitutively produced (i.e. no longer requires the environmental signal for expression). Although the origins of this concept can be traced to the latter half of the 19th century (Spalding, 1873; Baldwin, 1896; Morgan, 1896; Osborn, 1897), its formulation in a genetic context was done independently in the 1940s by Waddington (Waddington, 1942; Waddington, 1952; Waddington, 1953; Waddington, 1961) and Schmalhausen (Schmalhausen, 1949). All of these authors envisioned genetic assimilation as a means of facilitating phenotypic evolution. By 1953, however, G. G. Simpson had dismissed it: '... [genetic assimilation] is an interesting but, I would judge, relatively minor outcome of the [synthetic] theory' (Simpson, 1953), a sentiment to be echoed by other writers, e.g. 'It represents merely a degeneration of a part of an original adaptation' (Williams, 1966) and 'a baroque hypothesis' (Orr, 1999).

Despite such admonitions, interest in genetic assimilation continues to increase, with a variety of updated conceptual treatments (e.g. Rollo, 1994; Schlichting and Pigliucci, 1998; Pigliucci and Murren, 2003; Price et al., 2003; West-Eberhard, 2003; Schlichting, 2004; Badyaev, 2005). Recently, it has again been argued (de Jong, 2005) that genetic assimilation does not play an important role in evolution, and de Jong asserted that the proposed updates are conceptually flawed. Here, we examine her key criticisms:

(1) An emphasis on genetic assimilation denies the ecological importance of phenotypic plasticity.

(2) Quantitative genetic models are fully capable of predicting the adaptive role of phenotypic plasticity.

(3) There are no clear examples of genetic assimilation.

(4) Phenotypic plasticity does not represent an alternative to the Modern Synthesis.

To make sense of these arguments, and of why we consider that they miss the mark, we need first to briefly examine what phenotypic plasticity and genetic assimilation actually are. We will also need to briefly discuss the role(s) of quantitative genetics in evolutionary theory and practice, to address the charge that certain ideas about plasticity and assimilation are contradictory of established models in quantitative genetics.

Phenotypic plasticity and genetic assimilation: the basics

A fundamental issue about which there seems to be much disagreement concerns the role(s) of phenotypic plasticity [concepts and methods have recently been reviewed (Pigliucci, 2001)] and genetic assimilation (see below) within the context of modern evolutionary theory. 


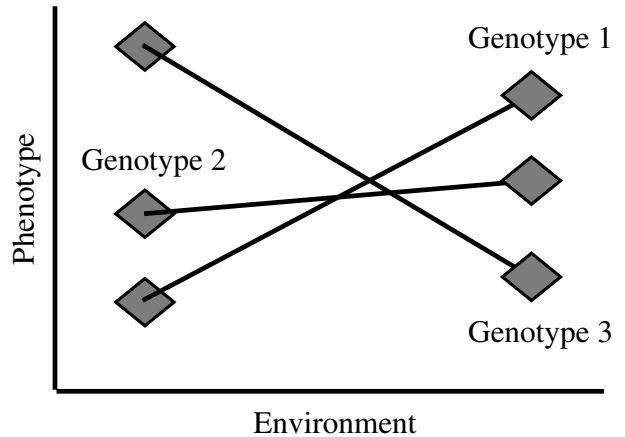

Fig. 1. An example of genotypic reaction norms illustrating the concept of phenotypic plasticity. In the simple case of two environments, the lines represent the norms of reaction of each genotype, while the slope is a measure of the degree and pattern (positive or negative) of phenotypic plasticity. So, for example, genotypes 1 and 3 are both plastic, but display opposite patterns in response to the same environments; genotype 2, on the other hand, shows little plasticity for this trait in this environmental set.

Phenotypic plasticity is usually defined as a property of individual genotypes to produce different phenotypes when exposed to different environmental conditions (Fig. 1). However, some important points need to be considered for the purposes of the present discussion. First, not all phenotypic plasticity is adaptive (in the evolutionary sense of improving the organism's survival or reproduction). Some traits are plastic because of unavoidable constraints imposed by the biochemistry, physiology or developmental biology of the organism (Sultan, 1995). Secondly, plasticity may be expressed at the behavioral, biochemical, physiological or developmental levels; while all these phenomena share the fundamental biological property of being part of the genotypespecific repertoire of environmentally induced phenotypes, there are significant differences in the degree of reversibility of different kinds of plasticity. Typically, biochemical and physiological responses can be reversed over short time scales, while developmental plasticity tends to be irreversible or takes longer to be reversed.

Thirdly, the type and degree of plasticity are specific to individual traits and environmental conditions; the same trait may be plastic in response to, say, changes in temperature, but not to nutrients, and a certain trait may be plastic in response to temperature while other traits are not. Finally, there seems to be abundant genetic variation for a variety of plastic responses in natural populations, which makes possible the evolution of plasticity by natural selection and other mechanisms.

As for genetic assimilation (GA), although it is Waddington's term that is used, it is Schmalhausen's conception (Schmalhausen, 1949) that is closer to our modern interpretation. Waddington's experiments began by showing that certain phenotypes [e.g. cross-veinless in Drosophila melanogaster (Waddington, 1953)] can be obtained at low frequency in a population by an environmental stimulus (e.g.
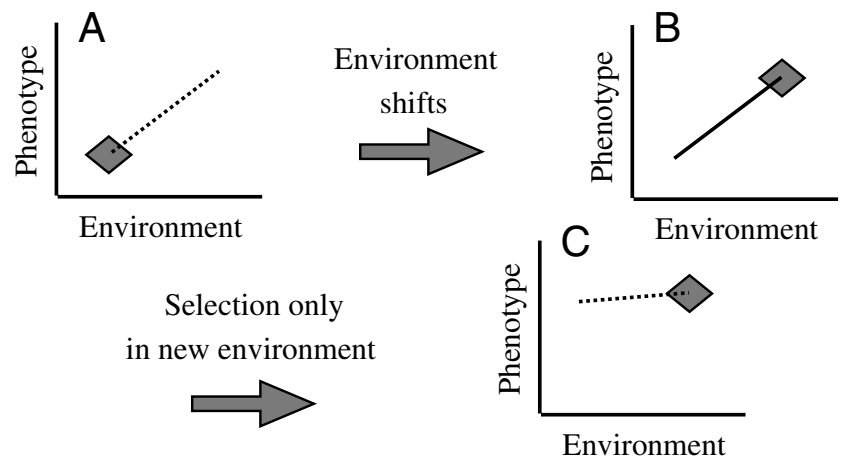

Fig. 2. The concept of genetic assimilation, seen in the modern light of reaction norms and phenotypic plasticity. The population is initially occupying one environment (A), although there is an unexpressed capacity for plasticity, should the environment change. If the environment does change (B), the pre-existing reaction norm allows the population to persist, producing a novel phenotype with no initial genetic change. Finally, if natural selection keeps operating only in the new environment $(\mathrm{C})$, the novel phenotype may become genetically fixed (assimilated), and the original reaction norm may lose plasticity, for example because of drift or costs associated with maintaining plasticity when it is not favored by natural selection (because the old environment is no longer experienced).

heat shock at certain stages of development). Today we would consider this a form of non-adaptive phenotypic plasticity induced by stress. Waddington went on to select flies to increase the frequency of the novel phenotype in response to the environmental stimulation. In other words, he was selecting for that form of phenotypic plasticity to become more frequent in the population (Fig. 2). After relatively few generations of selection he observed something unexpected: not only had the frequency of the novel phenotype reached very high levels, but the environmental stimulus no longer seemed necessary to elicit the appearance of the crossveinless phenotype! This apparent 'inheritance of an acquired character' was explained by Waddington in standard Darwinian terms as selection on the activation threshold for the trait in question during development. Waddington introduced the word 'assimilation' to refer to this outcome and discussed it in terms of canalization of the phenotype. Schmalhausen's perspective (Schmalhausen, 1949) was similar to the modern view: his 'stabilizing selection' involved the exposure of hidden variation by a new environmental stimulus, followed by selection for adaptive reactions to the stimulus, and finally stabilization of the reaction norm.

In summary, phenotypic plasticity is a common property of the reaction norm of a genotype (for a given trait, within a certain range of environmental conditions). Plasticity is what makes possible the appearance of an environmentally induced novel phenotype, and a process of selection on the expression of such phenotype in a new environment may end up 'fixing' (genetically assimilating) it by altering the shape of the reaction norm. 


\section{Recent proposals about the roles of plasticity and assimilation}

Before we can turn to an examination of the objections listed above, we need to have a better understanding of the substance against what exactly such objections have been raised.

Pigliucci and Murren make the claim that GA, long ignored as a phenomenon of possible interest to evolutionary theorists despite Waddington's experimental demonstration of its possibility, should be carefully reconsidered (Pigliucci and Murren, 2003). They pointed to a significant amount of circumstantial evidence that is compatible with partial or complete GA in several species of plants and animals. In our view, phenotypic plasticity could facilitate the expression of relatively well-adapted phenotypes under novel conditions (e.g. after migration to a new geographical area), and therefore allow a population to persist. Selection on the novel phenotype in the new environment would then simultaneously alter the reaction norm [e.g. because of costs associated with plasticity (Relyea, 2002; van Kleunen and Fischer, 2005)], and improve the performance of the population, resulting in the genetic assimilation of the trait in the new environment. This has the potential to explain a variety of evolutionary ecological processes, including, for example, the lag phase and successive population explosion of many invasive species.

West-Eberhard's claims about the role of plasticity in evolution are more sweeping (West-Eberhard, 2003), and result from a broad treatment of the general problem of phenotypic evolution (see also Schlichting and Pigliucci, 1998). West-Eberhard sees phenotypic evolution as the result of four steps:

(1) Trait origin: a mutation or environmental change causes the appearance of a developmental variant expressing a novel trait.

(2) Phenotypic accommodation (i.e. a rearrangement of different aspects of the phenotype) to the new trait, made possible by the inherent, pre-existing, plasticity of the developmental system.

(3) Initial spread of the new variant, facilitated by its recurrence in the population, if the initial change is environmental (in other words, one does not have to wait for a rare mutation to occur again before reaching relatively high frequency in the population).

(4) Genetic accommodation (i.e. fixation by allelic substitution) of the novel phenotype, as the result of standard selection.

The scenario proposed by Pigliucci and Murren for GA via evolution of plasticity (Pigliucci and Murren, 2003) would be a particular case of the broader possibility envisioned by WestEberhard, specifically when the origin of the trait (step 1) is due to an environmental, rather than a genetic, change. How prevalent the situations proposed by these authors (Pigliucci and Murren, 2003; West-Eberhard, 2003) actually are, is of course, a matter for empirical investigation.

Finally, several authors have pointed out that genetic assimilation may also have broad evolutionary consequences through the integration of different phenotypic characteristics
(Pigliucci and Preston, 2004). Assimilation may reveal, or even increase, the plasticity of traits correlated to the one being assimilated because - by making the regulation of one trait (e.g. an originally learnt behavior) independent of the presence of an environmental stimulus - another trait (e.g. a correlated behavior) can be conditionally expressed with much higher probability [the stretch-assimilate principle (see Jablonka and Lamb, 2005)].

\section{The critiques, and why they miss the mark}

We are now in a better position to understand the criticisms of recent work on the potential role of plasticity and assimilation in evolution.

(1) 'In genetic assimilation, phenotypic plasticity is not itself of importance, but only an intermediate stage to a new genetically fixed and phenotypically invariant state' [(de Jong, 2005), p101]. de Jong claims that a focus on GA diminishes the importance of phenotypic plasticity (PP) as an adaptive trait. We suggest that she misses the point. In GA, plasticity is of paramount importance because it allows the initial survival of the organism under novel environmental conditions. However, if the new conditions are the only ones being experienced by the population (i.e. the environment is not predictably variable), then standard evolutionary theory predicts the loss of plasticity and the evolution of a canalized phenotype: plasticity has led to assimilation. On the other hand, when both the old and new environments continue to be encountered, selection will favor the evolution of a reaction norm that is appropriately plastic.

(2) 'The adaptive role of phenotypic plasticity can be predicted from [quantitative genetic] models, not as a consequence of a developmental plasticity that is inherent to life and on a par with selection' [(de Jong, 2005), p113]. Let us deal with the second half of this statement first. To the best of our knowledge, no one has ever suggested that plasticity plays a role in evolution as a mechanism 'on a par with' natural selection. Indeed, this would be what philosophers of science refer to as a category mistake (as in asking "what is the color of triangles?'), since plasticity is a mechanism in the sense of a proximate cause of developmental (or biochemical, physiological, behavioral) changes, while natural selection is an ultimate cause of adaptation during evolution. Therefore, selection acts on developmental plasticity, and the two simply cannot be considered alternatives from a logical standpoint.

Returning to the efficacy of quantitative genetic models, we need to emphasize the distinction between two different roles of such models in evolutionary biology (e.g. Pigliucci and Schlichting, 1997). On one hand, quantitative genetics (QG) is a body of theory aimed at developing simple mathematical models, such as those presented by de Jong (de Jong, 2005), that can account in a general fashion for patterns of phenotypic evolution in natural populations. In this sense, there is no doubt that QG can 'explain' the evolution of phenotypic plasticity. However, many possible details of the specific mechanisms are equally compatible with any particular model, so that the 
explanatory power is somewhat weakened, and cannot reliably distinguish similar outcomes of different historical pathways of evolution.

In the case of de Jong's QG model (de Jong, 2005), her ability to claim that the evolution of ecotypes requires no expression of hidden plasticity derives from the assumption that trait values in different environmental states form a continuum. The result is that trait's expression becomes 'predictable' even in putatively novel environments (but see Schlichting and Pigliucci, 1998; Dudash et al., 2005).

The problem worsens if one turns to the other aspect of QG, i.e. the inference of possible causal mechanisms and/or historical paths, or the prediction of future outcomes. Its statistical tool set aims at summarizing the genetic variances and covariances among traits in a population, but while it is true that any study of phenotypic diversity in natural populations has to start with statistical summaries, it does not follow that such summaries can profitably be used for inferential purposes. When de Jong, for example, examines plots of reaction norms and their inter-environmental genetic correlations, deducing the presence or absence of physiological mechanisms underlying such patterns (de Jong, 2005), she is stepping far outside the reasonable boundaries for such methods. As Shipley, for one, has convincingly argued (Shipley, 2000), though variance-covariance patterns can be used to generate testable causal hypotheses, they most definitely cannot lead to trustworthy inferences about underlying mechanisms. The reason for this is - again - the well-known fact that many causal paths may lead to very similar phenotypic outcomes, and the latter cannot be used to go back to the former, no matter how clever and sophisticated the statistical tools. This is simply a fact of life for biologists and quantitative scientists in general, and it will not do to pretend otherwise.

Finally, with regard to the utility of QG models, we note that Price et al. have modeled the evolution of peak shifts via genetic assimilation using standard QG models (Price et al., 2003). In their simulations, various levels of plasticity were investigated: if plasticity was low, the population either went extinct or remained 'trapped' under the low peak; if plasticity was high, the peak shift could be accomplished directly via plasticity and no genetic change would be engendered. However, at intermediate levels of plasticity the phenotype produced moves into the attractive domain of the higher peak, and a period of constancy of this new environment leads to a peak shift via genetic assimilation.

(3) 'There is a good reason for that lack of attention [to genetic assimilation as an evolutionary mechanism]: the lack of convincing examples" [(de Jong, 2005), p. 16]. Similarly, genetic assimilation has been cavalierly dismissed on the ground of lack of evidence: 'unless and until there are hard data demonstrating the frequent occurrence of assimilation, evolutionists will rightly refuse to ground theories of adaptation on such a baroque hypothesis' (Orr, 1999). Pigliucci and Murren anticipated such criticism (Pigliucci and Murren, 2003), and warned that it makes little sense to deny the relevance of GA in evolution on the basis of a current dearth of evidence. First, the process may require only a few generations (as in Waddington's experiments), which means that it could occur so rapidly as to pass below the radar screen of evolutionary biologists, unless they were explicitly looking for it. Secondly, evolutionary biology is a historical science (Pigliucci, 2002), and in historical research 'evidence' is not simply out there for the taking, it becomes an object of a search in light of specific hypotheses (we would do well to remember Darwin's words in a letter to Henry Fawcett: 'How odd it is that anyone should not see that all observation must be for or against some view if it is to be of any service!' Numerous cases, in diverse organisms, have been identified that are compatible with the hypothesis of GA (see Rollo, 1994; Pigliucci and Murren, 2003; West-Eberhard, 2003; Tardieu, 1999; Chapman et al., 2000; Cooley et al., 2001; Sword, 2002; Price et al., 2003; Heil et al., 2004; Mery and Kawecki, 2004; Palmer, 2004; Keogh et al., 2005), so it seems that there is plenty of reasonable ground for advocating more explicit tests of the possibility that GA occurs in natural populations.

Additionally, since Pigliucci and Murren (Pigliucci and Murren, 2003) and Schlichting (Schlichting, 2004), several theoretical approaches continue to examine genetic assimilation under a variety of conditions (e.g. Wiles et al., 2005). Recent advances in the study of genetic assimilation include the use of computational models (Downing, 2004), and such models incorporate our current understanding of molecular biology (Behera and Nanjundiah, 2004). Other authors have taken a network modeling approach (Masel, 2004), or used the prisoner's dilemma framework (Suzuki and Arita, 2004). Together, these studies demonstrate that genetic assimilation is at least an active area of theoretical inquiry from a variety of perspectives.

Moreover, we would argue that, if the onus of evidence is to be placed on supporters of GA, we might reasonably expect that its detractors likewise demonstrate evidence for their favored schema. For example, how many times has the evolution of ecotypes (e.g. as proposed in de Jong's models) been observed in nature or the laboratory? For critics of GA as a facilitator of speciation, how many times has speciation been observed without an initial phase of plasticity or phenotypic accommodation?

(4) 'Phenotypic plasticity does not constitute a major alternative view of evolutionary biology, but takes its legitimate place in the neo-Darwinian modern synthesis' [(de Jong, 2005), p116]. de Jong's paper (de Jong, 2005) can be seen as an attempt to defend the Modern Synthesis [which she erroneously refers to as the 'neo-Darwinian' synthesis (Mayr and Provine, 1980)] from a perceived attack by the likes of West-Eberhard and the authors of the present paper. It is certainly true that several authors have pointed out limitations of the current paradigm in evolutionary biology, and have argued for inclusion of perspectives that have been ignored or downplayed in the past. Examples are ideas about punctuated equilibria, species selection, and the role of non-selective events in macroevolution (Gould, 2002); the elevation of environment to 


\section{M. Pigliucci, C. J. Murren and C. D. Schlichting}

an equal role with genes (Schlichting and Pigliucci, 1998; Lewontin, 2000); West-Eberhard's treatment of phenotypic accommodation (West-Eberhard, 2003); and most recently, Jablonka and Lamb's perspective on the importance of epigenetic inheritance systems (Jablonka and Lamb, 2005).

All of these, however, have clearly been intended as extensions of the Modern Synthesis, not rejections of it, just as the Synthesis itself has always been interpreted (correctly) as an extension, not a rejection, of Darwin's original insight that organismal history and diversification is largely a result of common descent and natural selection (Darwin, 1859). Indeed, evolutionary biology may be one of the most glaring exceptions to philosopher Thomas Kuhn's idea (Kuhn, 1970) that progress in science takes place through occasional revolutions (paradigm shifts), as in the transition between the Ptolemaic and the Copernican systems, or between the Newtonian and relativistic conceptions of the universe. Since Darwin's original idea was, as far as we can tell, essentially correct, it stands to reason that all the work of the Modern Synthesis, as well as all current attempts to improve on the latter, are best thought of as additional ramifications stemming out of the same base tree, not as plots to uproot the Darwinian construction.

\section{Concluding remarks}

We think that the new ideas about phenotypic plasticity's role in evolution, as well as the re-evaluation of concepts such as GA and phenotypic accommodation, represent not a threat to the Modern Synthesis, but rather a welcome expansion of its current horizon. Moreover, these ideas are generally compatible with current quantitative genetic models of phenotypic evolution (because the latter are largely invariant

$$
\begin{aligned}
& \text { Phenotypic } \\
& \text { plasticity }
\end{aligned}
$$

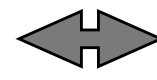

Developmental process

shaped by:

Natural

selection

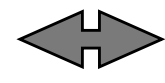

Evolutionary mechanism

results in:

Genetic assimilation or accommodation

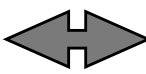

Evolutionary outcome

Fig. 3. The hierarchical/conceptual relationships among phenotypic plasticity, natural selection, and genetic assimilation or phenotypic accommodation. Plasticity is a developmental process on which the evolutionary mechanism of selection may operate, yielding the evolutionary outcome (under certain conditions) of assimilation/ accommodation. Seen this way, there is no possibility of confusing plasticity as ontologically equivalent to natural selection, and therefore somehow a threat to the Modern Synthesis. with respect to specific mechanisms), and offer the potential for a fruitful empirical research program that need not be prematurely quashed due to superficial critiques.

However, we recognize that the criticisms raised by de Jong are just one example of a relatively common attitude among evolutionary biologists (de Jong, 2005), and we think the reason for this is some persistent level of conceptual confusion about the proper domains of terms such as natural selection, phenotypic plasticity, and genetic assimilation. Fig. 3 shows how these are actually related: phenotypic plasticity is (in part) a developmental process, not an evolutionary one. As such, it can be the target of natural selection (an evolutionary mechanism, though of course not the only one), and yield under certain conditions - the evolutionary outcome of genetic assimilation or phenotypic accommodation. Once one recognizes the clear hierarchical distinctions among these concepts, most fears about an imminent overthrow of the Modern Synthesis should dissipate.

We thank James Fordyce, Theodore Garland and Trevor Price for their helpful feedback, and Josh Banta for reading a previous draft of the manuscript.

\section{References}

Badyaev, A. V. (2005). Stress-induced variation in evolution: from behavioural plasticity to genetic assimilation. Proc. R. Soc. Lond. B Biol. Sci. 272, 877-886.

Baldwin, J. M. (1896). A new factor in evolution. Am. Nat. 30, 441-451; 536553.

Behera, N. and Nanjundiah, V. (2004). Phenotypic plasticity can potentiate rapid evolutionary change. J. Theor. Biol. 226, 177-184.

Cooley, J. R., Simon, C., Marshall, D. C., Slon, K. and Ehrhardt, C. (2001). Allochronic speciation, secondary contact, and reproductive character displacement in periodical cicadas (Hemiptera: Magicicada spp.): genetic, morphological, and behavioural evidence. Mol. Ecol. 10, 661-671.

Darwin, C. R. (1859). The Origin of Species by means of Natural Selection: or, the preservation of favored races in the struggle for life. New York: A. L. Burt (1910).

de Jong, G. (2005). Evolution of phenotypic plasticity: patterns of plasticity and the emergence of ecotypes. New Phytol. 166, 101-118.

Downing, K. L. (2004). Development and the Baldwin effect. Artif. Life 10, 39-63.

Dudash, M. R., Murren, C. J. and Carr, D. E. (2005). Using Mimulus as a model system to understand the role of inbreeding in conservation: genetic and ecological approaches. Ann. Mo. Bot. Gard. 92, 36-51.

Gould, S. J. (2002). The Structure of Evolutionary Theory. Cambridge, MA: Harvard University Press.

Heil, M., Greiner, S., Meimberg, H., Kruger, R., Noyer, J.-L., Heubl, G., Linsenmair, K. E. and Boland, W. (2004). Evolutionary change from induced to constitutive expression of an indirect plant resistance. Nature 430, 205-208.

Jablonka, E. and Lamb, M. J. (2005). Evolution in Four Dimensions: Genetic, Epigenetic, Behavioral, and Symbolic Variation in the History of Life. Cambridge, MA: MIT Press.

Keogh, J. S., Scott, I. A. W. and Hayes, C. (2005). Rapid and repeated origin of insular gigantism and dwarfism in Australian tiger snakes. Evolution 59, 226-233.

Kuhn, T. (1970). The Structure of Scientific Revolutions. Chicago, IL: University of Chicago Press.

Lewontin, R. C. (2000). The Triple Helix. Cambridge, MA: Harvard University Press.

Masel, J. (2004). Genetic assimilation can occur in the absence of selection for the assimilating phenotype, suggesting a role for the canalization heuristic. J. Evol. Biol. 17, 1106-1110. 
Mayr, E. and Provine, W. B. (1980). The Evolutionary Synthesis. Cambridge, MA: Harvard University Press.

Mery, F. and Kawecki, T. J. (2004). The effect of learning on experimental evolution of resource preference in Drosophila melanogaster. Evolution 58, 757-767.

Morgan, C. L. (1896). Habit and Instinct. London: Arnold.

Orr, H. A. (1999). An evolutionary dead end? Science 285, 343-344.

Osborn, H. F. (1897). Organic selection. Science 15, 583-587.

Palmer, A. R. (2004). Symmetry breaking and the evolution of development. Science 306, 828-833.

Pigliucci, M. (2001). Phenotypic Plasticity: Beyond Nature and Nurture. Baltimore, MD: Johns Hopkins University Press.

Pigliucci, M. (2002). Are ecology and evolutionary biology 'soft' sciences? Ann. Zool. Fenn. 39, 87-98.

Pigliucci, M. and Murren, C. J. (2003). Genetic assimilation and a possible evolutionary paradox: can macroevolution sometimes be so fast as to pass us by? Evolution 57, 1455-1464.

Pigliucci, M. and Schlichting, C. D. (1997). On the limits of quantitative genetics for the study of phenotypic evolution. Acta Biotheor. 45, 143-160.

Pigliucci, M. and Schmitt, J. (2004). Phenotypic plasticity in response to foliar and neutral shade in gibberellin mutants of Arabidopsis thaliana. Evol. Ecol. Res. 6, 243-249.

Price, T. D., Qvarnstrom, A. and Irwin, D. E. (2003). The role of phenotypic plasticity in driving genetic evolution. Proc. R. Soc. Lond. B Biol. Sci. 270, $1433-1440$

Relyea, R. A. (2002). Costs of phenotypic plasticity. Am. Nat. 159, 272-282.

Rollo, C. D. (1994). Phenotypes: Their Epigenetics, Ecology and Evolution. London: Chapman \& Hall.

Schlichting, C. D. (1986). The evolution of phenotypic plasticity in plants. Annu. Rev. Ecol. Syst. 17, 667-693.

Schlichting, C. D. (2004). The role of phenotypic plasticity in diversification. In Phenotypic Plasticity: Functional and Conceptual Approaches (ed. T. J. DeWitt and S. M. Scheiner), pp. 191-200. Oxford: Oxford University Press.

Schlichting, C. D. and Pigliucci, M. (1998). Phenotypic Evolution: A Reaction Norm Perspective. Sunderland, MA: Sinauer Associates.

Schmalhausen, I. I. (1949). Factors of Evolution. Philadelphia, PA: Blakiston. Shipley, B. (2000). Cause and Correlation in Biology: A User's Guide to Path
Analysis, Structural Equations and Causal Inference. Cambridge: Cambridge University Press.

Simpson, G. G. (1953). The Baldwin effect. Evolution 7, 110-117.

Smith, H. (1982). Light quality, photoreception, and plant strategy. Annu. Rev. Plant Physiol. 33, 481-518.

Spalding, D. (1873). Instinct with original observations on young animals. Macmillan's Magazine 27, 282-293.

Sultan, S. E. (1987). Evolutionary implications of phenotypic plasticity in plants. Evol. Biol. 21, 127-178.

Sultan, S. E. (1995). Phenotypic plasticity and plant adaptation. Acta Bot. Neerl. 44, 363-383.

Suzuki, R. and Arita, T. (2004). Interactions between learning and evolution: the outstanding strategy generated by the Baldwin effect. Biosystems 77, 5771.

Sword, G. A. (2002). A role for phenotypic plasticity in the evolution of aposematism. Proc. R. Soc. Lond. B Biol. Sci. 1501, 1639-1644.

Tardieu, C. (1999). Ontogeny and phylogeny of femoro-tibial characters in humans and hominid fossils: functional influence and genetic determinism. Am. J. Phys. Anthropol. 110, 365-377.

van Kleunen, M. and Fischer, M. (2005). Constraints on the evolution of adaptive phenotypic plasticity in plants. New Phytol. 165, 49-60.

Via, S. (1993). Adaptive phenotypic plasticity: target or by-product of selection in a variable environment? Am. Nat. 142, 352-365.

Waddington, C. H. (1942). Canalization of development and the inheritance of acquired characters. Nature 150, 563-565.

Waddington, C. H. (1952). Selection of the genetic basis for an acquired character. Nature 169, 278.

Waddington, C. H. (1953). Genetic assimilation of an acquired character. Evolution 7, 118-126.

Waddington, C. H. (1961). Genetic assimilation. Adv. Genet. 10, 257-290.

West-Eberhard, M. J. (2003). Developmental Plasticity and Evolution. New York: Oxford University Press.

Wiles, J., Watson, J., Tonkes, B. and Deacon, T. (2005). Transient phenomena in learning and evolution: genetic assimilation and genetic redistribution. Artif. Life 11, 177-188.

Williams, G. C. (1966). Adaptation and Natural Selection. Princeton, NJ: Princeton University Press. 